PROCEEDINGS OF THE

AMERICAN MATHEMATICAL SOCIETY

Volume 127, Number 10, Pages 2883-2887

S 0002-9939(99)05265-X

Article electronically published on April 23, 1999

\title{
EQUIVALENCE OF A $K$-FUNCTIONAL WITH THE APPROXIMATION BEHAVIOR OF SOME LINEAR MEANS FOR ABSTRACT FOURIER SERIES
}

\author{
WALTER TREBELS
}

(Communicated by Frederick W. Gehring)

\begin{abstract}
Within the setting of abstract Cesàro-bounded Fourier series a $K$-functional is introduced and characterized by the convergence behavior of some linear means. Applications are given within the framework of Jacobi, Laguerre and Hermite expansions. In particular, Ditzian's (1996) equivalence result in the setting of Legendre expansions is covered.
\end{abstract}

\section{INTRODUCTION}

In [3] Ditzian considers the $K$-functional

$$
K(f, t)_{p}=\inf _{g \in C^{2}[-1,1]}\left(\|f-g\|_{p}+t\left\|\frac{d}{d x}\left(1-x^{2}\right) \frac{d}{d x} g\right\|_{p}\right),
$$

where $\|\cdot\|_{p}=\|\cdot\|_{L^{p}(-1,1)}$, and characterizes it by

$$
K\left(f, n^{-2}\right)_{p} \approx\left\|R_{n} f-f\right\|_{p}, \quad R_{n} f=\sum_{k=0}^{n}\left(1-\frac{k(k+1)}{n(n+1)}\right) a_{k} P_{k} .
$$

The $P_{k}$ 's are the Legendre polynomials, the $a_{k}$ 's the Fourier-Legendre coefficients of $f$, and $A_{n} \approx B_{n}$ means that there is a constant $C$ such that $C^{-1} A_{n} \leq B_{n} \leq C A_{n}$. Generic positive constants that are independent of functions $f$ and parameters $n$ will be denoted by $C$.

Ditzian's proof of the above result makes essential use of an explicit relation between the special approximation process $R_{n}$ and the differential operator in (1). Now note that the involved differential operator basically determines the saturation class of the corresponding approximaton process (see [2, II]). This observation is the reason for the validity of an equivalence result in the setting of abstract Cesàrobounded orthogonal expansions. Its short proof is based on the two algebraic identities (5), (6), and the elementary multiplier criterion Theorem A.

Received by the editors December 3, 1997.

1991 Mathematics Subject Classification. Primary 41A65, 41A40, 33C45.

Key words and phrases. $K$-functional, linear approximation processes, saturation, orthogonal expansions. 
Applications are given within the framework of Jacobi, Laguerre and Hermite expansions. The method also applies to integral transforms whose inverse are Rieszsummable and gives analogous results. Such integral transforms are, e.g., the (modified) Hankel transform and the multivariate Fourier transform - for the latter we require that the involved differential operator have a (quasi-) radial symbol.

Let us recall $(a)$ the concept of a Fourier series in a Banach space $X$ from Butzer, Nessel, and Trebels [2] and (b) a sufficient multiplier criterion from [7] in the case that this Fourier series is Cesàro bounded.

(a) Let $\left\{f_{n}^{*}, f_{n}\right\}_{n=0}^{\infty},\left\{f_{n}\right\} \subset X,\left\{f_{n}^{*}\right\} \subset X^{*}$ be a complete, fundamental, biorthogonal system, i.e., $(i)$ if $f \in X$, then $f_{k}^{*}(f)=0$ for all $k \in \mathbf{N}_{0}$ implies $f=0$, (ii) the linear span $F$ of the $f_{k}$ 's is dense in $X, \bar{F}^{X}=X$, and $(i i i) f_{k}^{*}\left(f_{j}\right)=0$ for all $j \neq k \in N_{0}$. We associate to $f \in X$ its Fourier series and then formally to each scalar-valued sequence $\left\{m_{k}\right\}$ an operator $T_{m}$ by

$$
f \sim \sum_{k=0}^{\infty} h_{k} f_{k}^{*}(f) f_{k}, \quad T_{m} f \sim \sum_{k=0}^{\infty} h_{k} m_{k} f_{k}^{*}(f) f_{k}, \quad h_{k}=1 / f_{k}^{*}\left(f_{k}\right) ;
$$

equality holds for $f \in F$. If for some constant $C>0$, independent of $f,\left\|T_{m} f\right\| \leq$ $C\|f\|$ is valid for all $f \in F$, we call $m=\left\{m_{k}\right\}$ a bounded multiplier and define its multiplier norm $\left\|\left\{m_{k}\right\}\right\|_{M}$ to be the operator norm.

(b) We say the pair $\left(X,\left\{f_{k}^{*}, f_{k}\right\}\right)$ is $(C, \delta)$-bounded, $\delta \geq 0$, if for all elements $f \in F$ the Cesàro means $(C, \delta)_{n} f$ are uniformly bounded, i.e.,

$$
\left\|(C, \delta)_{n} f\right\| \leq C\|f\|, \quad(C, \delta)_{n} f=\sum_{k=0}^{n} \frac{A_{n-k}^{\delta}}{A_{n}^{\delta}} h_{k} f_{k}^{*}(f) f_{k}, A_{n}^{\delta}=\frac{\Gamma(n+\delta+1)}{\Gamma(n+1) \Gamma(\delta+1)} .
$$

Then it is well known that all $\left(C, \delta^{\prime}\right)$-means with $\delta^{\prime}>\delta$ are also uniformly bounded. The Cesàro boundedness of $\left(X,\left\{f_{k}^{*}, f_{k}\right\}\right)$ implies the following useful sufficient multiplier criterion.

Theorem A ([7, Theorem 3.9]). Let $\left(X,\left\{f_{k}^{*}, f_{k}\right\}\right)$ be $(C, \delta)$-bounded; choose a $\in$ $\mathbf{N}$ with $a-1<\delta \leq a$.

i) Define normed subspaces $B V_{a+1}$ of $C_{0}[0, \infty)$, the set of continuous functions vanishing at infinity, by

$$
B V_{a+1}=\left\{m \in C_{0}: m, \ldots, m^{(a-1)} \in A C_{\mathrm{loc}}, m^{(a)} \in B V_{\mathrm{loc}},\|m\|_{B V_{a+1}}<\infty\right\},
$$

where $\|m\|_{B V_{a+1}}=\int_{0}^{\infty} t^{a}\left|d m^{(a)}(t)\right|$.

ii) Let $\Phi$ be a non-negative, strictly increasing function with $\lim _{t \rightarrow 0+} \Phi(t)=0$ and $\lim _{t \rightarrow \infty} \Phi(t)=\infty$, and let $\Phi$ possess $(a+1)$ continuous derivatives on $(0, \infty)$ with

$$
\left|t^{k} \Phi^{(k+1)}(t)\right| \leq D \Phi^{\prime}(t), \quad t>0, \quad 0 \leq k \leq a .
$$

iii) Let $\Psi$ be a positive, increasing function on $(0, \infty)$.

Then, for each $m \in B V_{a+1}$, there holds uniformly in $\rho$

$$
\left\|\{m(\Phi(k) / \Psi(\rho))\}_{k}\right\|_{M} \leq C^{*}\|m\|_{B V_{a+1}} .
$$

In particular, $\Phi(t)=\Psi(t)=(t(t+c))^{\gamma}, c \geq 0, \gamma>0$, are admitted so that in the proof of Theorem 1 below, without loss of generality, we may restrict ourselves to the case $\gamma=1$. 


\section{The Equivalence Result}

Theorem 1. Let $\left(X,\left\{f_{k}^{*}, f_{k}\right\}\right)$ be $(C, \delta)$-bounded; let $D: F \rightarrow X$ be a linear operator defined by $D f_{k}=\Phi(k) f_{k}$ for all $k \in \mathbf{N}_{0}, \Phi$ as in Theorem $A$, and (fractional) powers $D^{\gamma}, \gamma>0$, of $D$ by $D^{\gamma} f_{k}=(\Phi(k))^{\gamma} f_{k}$. Define on $X$ the $K_{\gamma}$-functional

$$
K_{\gamma}\left(f, t^{\gamma}\right)=\inf _{g \in F}\left(\|f-g\|+t^{\gamma}\left\|D^{\gamma} g\right\|\right) .
$$

Then for $a \in \mathbf{N}, a \geq \delta$, we have

$$
K_{\gamma}\left(f,(\Phi(n))^{-\gamma}\right) \approx\left\|T_{\Phi_{n}^{\gamma}} f-f\right\|, \quad T_{\Phi_{n}^{\gamma}} f=\sum_{k=0}^{n}\left(1-\frac{(\Phi(k))^{\gamma}}{(\Phi(n))^{\gamma}}\right)^{a} h_{k} f_{k}^{*}(f) f_{k} .
$$

Remark. The approximation process $T_{\Phi_{n}^{\gamma}}$ can of course be replaced by any equivalent approximation process (see [2, I] and [7]); thus, e.g., the exponent $a$ in (4) may be replaced by any $\delta^{\prime} \geq \delta$, or the "polynomial" means $T_{\Phi_{n}^{\gamma}}$ by Gauss-Weierstrass type means like

$$
W_{\gamma, \Phi(n)}(f)=\sum_{k=0}^{\infty} e^{-(\Phi(k) / \Phi(n))^{\gamma}} h_{k} f_{k}^{*}(f) f_{k} .
$$

The comparison results in [2, I] and [7] (also derived by the multiplier method below) imply results of the type $K_{\gamma^{\prime}}\left(f, t^{\gamma^{\prime}}\right) \leq C K_{\gamma}\left(f, t^{\gamma}\right), \gamma^{\prime}>\gamma$.

The Proof of Theorem 1 is a combination of an elementary multiplier argument (based on Theorem A) with the standard procedure one follows when estimating $K$-functionals. First observe that $m(t)=(1-t)_{+}^{a} \in B V_{a+1}$; here we use the notation $(1-t)_{+}=1-t$ if $t<1$ and $=0$ otherwise. Thus, by Theorem $\mathrm{A}, T_{\Phi_{n}^{\gamma}}$ is a uniformly bounded family of linear operators with $T_{\Phi_{n}^{\gamma}} f \rightarrow f$ for all $f \in F$ if $n \rightarrow \infty$; hence $T_{\Phi_{n}^{\gamma}}$ defines an approximation process on $X$. As mentioned above, without loss of generality, we may restrict ourselves to the case $\gamma=1$ and then write $K(f, t)=K_{1}(f, t), \Phi_{n}=\Phi_{n}^{1}$.

Let us first estimate the $K$-functional. Obviously,

$$
K\left(f,(\Phi(n))^{-1}\right) \leq\left\|f-T_{\Phi_{n}} f\right\|+(\Phi(n))^{-1}\left\|D T_{\Phi_{n}} f\right\| .
$$

For the Fourier coefficients of the last term there holds

$$
(\Phi(n))^{-1} f_{k}^{*}\left(D T_{\Phi_{n}} f\right)=\frac{(\Phi(k) / \Phi(n))(1-\Phi(k) / \Phi(n))_{+}^{a}}{1-(1-\Phi(k) / \Phi(n))_{+}^{a}} f_{k}^{*}\left(f-T_{\Phi_{n}} f\right) .
$$

Now $m(t)=t(1-t)_{+}^{a} /\left(1-(1-t)_{+}^{a}\right) \in B V_{a+1}$. Thus, Theorem A shows that the (quotient) sequence on the right side generates a uniformly bounded family of linear operators $M_{n}$. By the completeness of the biorthogonal system $\left\{f_{k}^{*}, f_{k}\right\}$

$$
(\Phi(n))^{-1} D T_{\Phi_{n}} f=M_{n}\left(f-T_{\Phi_{n}} f\right),
$$

whence $K\left(f,(\Phi(n))^{-1}\right) \leq C\left\|f-T_{\Phi_{n}} f\right\|$.

To show the converse inequality choose $g \in F$ such that

$$
\|f-g\|+(\Phi(n))^{-1}\|D g\| \leq 2 K\left(f,(\Phi(n))^{-1}\right) .
$$

Then

$$
\left\|f-T_{\Phi_{n}} f\right\| \leq\left\|(f-g)-T_{\Phi_{n}}(f-g)\right\|+\left\|g-T_{\Phi_{n}} g\right\| .
$$


From the Minkowski inequality and the argument at the beginning of the proof, the first term of the right side is dominated by $C\|f-g\|$; we have only to discuss the second one. Obviously,

$$
f_{k}^{*}\left(g-T_{\Phi_{n}} g\right)=\frac{1-(1-\Phi(k) / \Phi(n))_{+}^{a}}{\Phi(k) / \Phi(n)} \frac{1}{\Phi(n)} f_{k}^{*}(D g) .
$$

In $\left[2\right.$, p. 563] it is verified directly that $m(t)=\left(1-(1-t)_{+}^{a}\right) / t \in B V_{a+1}$. Therefore, by Theorem A, the corresponding (quotient) sequence preceding $(\Phi(n))^{-1} f_{k}^{*}(D g)$ generates a uniformly bounded operator family $M_{n}$. Thus

$$
g-T_{\Phi_{n}} g=(\Phi(n))^{-1} M_{n}(D g),
$$

and, by the choice of $g$, the assertion

$$
\left\|f-T_{\Phi_{n}} f\right\| \leq C\|f-g\|+C(\Phi(n))^{-1}\|D g\| \leq C K(f, 1 / \Phi(n)) .
$$

\section{Applications}

a) Jacobi expansions. Choose $X=L^{p}\left((-1,1),(1-x)^{\alpha}(1+x)^{\beta} d x\right), 1 \leq p \leq$ $\infty, \alpha \geq \beta \geq-1 / 2$; of course one has here - analogously in the applications below - to replace $L^{\infty}$ by $\bar{F}^{C}=C[-1,1]$, where $F$ is the span of the $f_{n}=P_{n}^{(\alpha, \beta)}$, the Jacobi polynomials, defined by

$$
\begin{aligned}
& (1-x)^{\alpha}(1+x)^{\beta} P_{n}^{(\alpha, \beta)}(x) \\
& \quad=\frac{(-1)^{n}}{2^{n} n !}\left(\frac{d}{d x}\right)^{n}\left((1-x)^{n+\alpha}(1+x)^{n+\beta}\right), x \in[-1,1], n \in \mathbf{N}_{0} .
\end{aligned}
$$

Define Fourier-Jacobi coefficients by $f_{k}^{*}(f)=\int_{-1}^{1} f(x) P_{n}^{(\alpha, \beta)}(x)(1-x)^{\alpha}(1+x)^{\beta} d x$. It was shown by Gasper [4] in combination with a result in [8, p. 246] that the Cesàro means of order $\delta>\alpha+1 / 2$ are uniformly bounded on $X$. When

$$
D=-\left(1-x^{2}\right) \frac{d^{2}}{d x^{2}}+(\alpha-\beta+(\alpha+\beta+2) x) \frac{d}{d x},
$$

then there holds (see $[8$, p. 60])

$$
D P_{n}^{(\alpha, \beta)}(x)=n(n+\alpha+\beta+1) P_{n}^{(\alpha, \beta)}(x) .
$$

For $\alpha=\beta=0$ one obtains $\Phi(n)=n(n+1)$ and the result of Ditzian [3] is regained by Theorem 1. Otherwise one obtains a characterization of the $K$-functional in weighted $L^{p}$-spaces. If $\alpha=\beta=-1 / 2$, one considers cosine expansions; then $(x=\cos t)$ we have $D=-d^{2} / d t^{2}$. We mention that in this situation $D^{1 / 2}$ has the classical interpretation $D^{1 / 2}=\frac{d}{d t} H$ with $H$ being the periodic Hilbert transform (see $[1$, p. 350]).

b) Laguerre expansions. Let the Laguerre polynomials $L_{n}^{\alpha}$ be given by

$$
n ! L_{n}^{\alpha}(x)=x^{-\alpha} e^{x}(d / d x)^{n}\left(x^{\alpha+n} e^{-x}\right), \quad x>0, n \in \mathbf{N}_{0} .
$$

Choose $\left\{f_{n}\right\}$ to be the system of the Laguerre functions

$$
\ell_{n}^{\alpha}(x)=(n ! / \Gamma(n+\alpha+1))^{1 / 2} e^{-x / 2} L_{n}^{\alpha}(x), \alpha \geq 0,
$$

orthonormalized in $L^{2}\left((0, \infty), x^{\alpha} d x\right)$. Defining the Fourier-Laguerre coefficients on $X=L^{p}\left((0, \infty), x^{\alpha} d x\right), 1 \leq p \leq \infty$, in the standard way by $f_{n}^{*}(f)=$ $\int_{0}^{\infty} f(x) \ell_{n}^{\alpha}(x) d x$ then, according to Görlich and Markett [5], the $(C, \delta)$-means are 
uniformly bounded for $\delta>\alpha+1 / 2$. Further we note that the $\ell_{n}^{\alpha}$ 's are eigenfunctions of the differential operator (see $[8$, p. 100])

$$
D_{\alpha}=-x \frac{d^{2}}{d x^{2}}+(\alpha+1-x) \frac{d}{d x}-\left(\frac{x}{4}+\frac{\alpha+1}{2}\right), \quad D_{\alpha} \ell_{n}^{\alpha}=n \ell_{n}^{\alpha} .
$$

Thus Theorem 1 gives a characterization of the corresponding $K$-functional in weighted $L^{p}\left(\mathbf{R}_{+}\right)$-spaces.

c) Hermite expansions. Let the Hermite polynomials $H_{n}$ be given by $H_{n}(x)=$ $(-1)^{n} \exp \left(x^{2}\right)(d / d x)^{n} \exp \left(-x^{2}\right)$. Choose $\left\{f_{n}\right\}$ to be the system of the Hermite functions

$$
\varphi_{n}(x)=\left(2^{n} \sqrt{\pi} n !\right)^{-1 / 2} e^{-x^{2} / 2} H_{n}(x),
$$

orthonormalized in $L^{2}(\mathbf{R}, d x)$. Define $f_{n}^{*}(f)=\int f(x) \varphi_{n}(x) d x$ on $L^{p}(\mathbf{R}, d x), 1 \leq$ $p \leq \infty$, as Fourier-Hermite coefficients. It has been proved by Thangavelu [6] that the corresponding Cesàro means are uniformly bounded for $\delta>1 / 6$. Since the $\varphi_{n}$ 's are eigenfunctions of the differential operator (see $[8$, p. 106])

$$
D=-\frac{d^{2}}{d x^{2}}+\left(x^{2}-1\right), \quad D \varphi_{n}(x)=2 n \varphi_{n}(x),
$$

Theorem 1 characterizes the corresponding $K$-functional.

\section{REFERENCES}

[1] P.L. Butzer and R.J. Nessel, Fourier-Analysis and Approximation, Birkhäuser, Basel, 1971. MR 58:23312

[2] P.L. Butzer, R.J. Nessel, and W. Trebels, On summation processes of Fourier expansions in Banach spaces. I Comparison theorems; II Saturation theorems, Tohôku Math. J. 24 (1972), 127 - 140; 551 - 569. MR 48:9196; MR 48:9197

[3] Z. Ditzian, A K-functional and the rate of convergence of some linear polynomial operators, Proc. Amer. Math. Soc. 124 (1996), 1773 - 1781. MR 96h:41026

[4] G. Gasper, Positivity and the convolution structure for Jacobi series, Ann. of Math. 93 (1971), 112 - 118. MR 44:1852

[5] E. Görlich and C. Markett, A convolution structure for Laguerre series, Indag. Math. 44 (1982), 161 - 171. MR 83g:42015

[6] S. Thangavelu, Summability of Hermite expansions I, Trans. Amer. Math. Soc. 314 (1989), 119 - 142. MR 91b:42048

[7] W. Trebels, Multipliers for $(C, \alpha)$-bounded Fourier Expansions in Banach Spaces and Approximation Theory, Lecture Notes in Math. vol. 329, Springer - Verlag, 1973. MR 58:23307

[8] G. Szegö, Orthogonal Polynomials, 4th ed., Amer. Math. Soc. Colloq. Publ. 23, Providence, R.I., 1975. MR 51:8724

Fachbereich Mathematik, Technische Universität Darmstadt, Schlossgartenstr. 7, D-64289 Darmstadt, Germany

E-mail address: trebels@mathematik.tu-darmstadt.de 